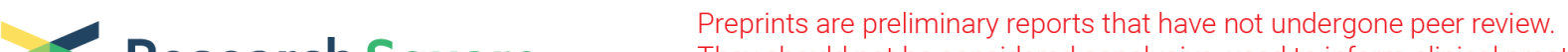

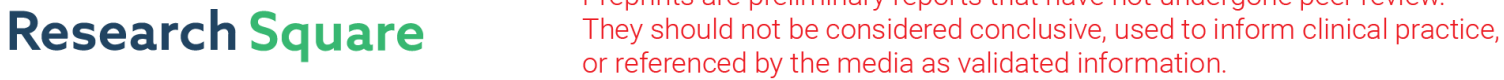 \\ PTPRZ1 Inhibits the Cisplatin Resistance of Ovarian Cancer by Regulating PI3K/AKT/mTOR Signal Pathway
}

\section{Peng Wang}

1.Qilu Hospital, Cheeloo College of Medicine, Shandong University

\section{Yuanjing $\mathrm{Hu}$}

Tianjin Central Hospital of Obstetrics and Gynecology

\section{Pengpeng Qu}

Tianjin Central Hospital of Obstetrics and Gynecology

\section{Ying Zhao}

Qilu Hospital, Cheeloo College of Medicind, Shandong University

Jing Liu

Tianjin Central Hospital of Obstetrics and Gynecology

Jianguo Zhao

Tianjin Central Hospital of Obstetrics and Gynecology

\section{Beihua Kong ( $\sim$ kongbeihuaqilu@163.com )}

Qilu Hospital, Cheeloo Cpllege Medicine, Shandong University https://orcid.org/0000-0002-8731-6643

\section{Research}

Keywords: Ovarian cancer, PTPRZ1, Cisplatin, PI3K/AKT/mTOR

Posted Date: August 12th, 2020

DOl: https://doi.org/10.21203/rs.3.rs-57236/v1

License: (c) (1) This work is licensed under a Creative Commons Attribution 4.0 International License. Read Full License 


\section{Abstract}

Background: Cisplatin resistance (DDP resistance) is a major cause for poor prognosis of ovarian cancer patients. PTPRZ1 has been proven to participate in the occurrence and development of multiple tumors, including tumor resistance. This study was designed to investigate the roles of PTPRZ1 in DDP-resistant ovarian cancer cells and their possible mechanism.

Methods: PTPRZ1 expression in ovarian cancer tissues and normal tissues was analyzed by GEPIA database and verified by qRT-PCR. PTPRZ1 expression in normal ovarian cancer cells and DDP-resistant ovarian cancer cells was also analyzed. Subsequently, qRT-PCR, western blot, MTT experiment and flow cytometry were used to assess the effects of PTPRZ1-PI3K/AKT/mTOR regulating axis on cisplatin resistance of ovarian cancer.

Results: PTPRZ1 expression was abnormally low in ovarian cancer tissues, and notably reduced in DDPresistant ovarian cancer cells. MTT experiment and flow cytometer indicated that overexpression of PTPRZ1 enhanced the cisplatin sensitivity of ovarian cancer cells and promoted the cell apoptosis. The results of mechanism research showed that PTPRZ1 exerted its biological effects possibly through blocking PI3K/AKT/mTOR pathway.

Conclusion: PTPRZ1 suppresses the cisplatin resistance of ovarian cancer and induces the cytotoxicity by blocking PI3K/AKT/mTOR pathway.

\section{Introduction}

Ovarian cancer $(\mathrm{OC})$ is a leading death cause of all gynecological malignant tumors, while epithelial ovarian cancer (EOC) is the most common OC(1). With the development of laparoscopic surgery, robot assisted surgery and adjuvant chemotherapy for $\mathrm{OC}$, the perioperative effects and prognosis of some $\mathrm{OC}$ patients are improved. However, as a result of toxic and side effects and drug resistance of chemotherapeutic drugs, the chemoresistance, tumor recurrence, extensive metastasis occur in most cases, and finally result in patient death $(2,3)$. The 5 -year survival rate of advanced OC is less than $30 \%$ now(4). It is very difficult to treat drug resistant OC patients and their quality of life is greatly affected.

The fist-line therapeutic regimens of $\mathrm{OC}$ include complete cytoreductive surgery and platinum- (cisplatin or carboplatin) and taxane- (paclitaxel) based first-line combined chemotherapy $(5,6)$. Many patients, especially uncontrolled or early recurrence patients, are vulnerable to progression into platinum resistance, thereby affecting their survival $(7,8)$. Therefore, platinum resistance has become an obstacle for chemotherapy and an important clinical challenge. Until now, there are very limited measures to prevent or reverse platinum resistance.

PTPRZ1 is mainly expressed in central nervous system, identified as a key factor for the recovery of demyelination damage, and is abnormally expressed in multiple tumors recently. For instance: PTPRZ1 is considered as an oncogene for promoting tumor growth in glioma that further results in the malignant 
progression of glioma by fusion with MET cancer gene(9); in breast cancer, PTPRZ1 reduces the chemosensitivity through promoting tumor cell growth and suppressing cell apoptosis(10); the proliferation of RCC cells enhanced by PTPRZ1 is dependent on the inactivation of VHL, while PTPRZ1/ $\beta$ catenin pathway may be a potential target for the treatment of non-active VHL RCC(11).

We observed that PTPRZ1 expression was considerably reduced in OC through bioinformatic analysis and qRT-PCR detection. Meanwhile, further detection revealed that its expression in cisplatin resistant cell lines was lower than that in normal cell lines. Therefore, with this point, we initially explored the roles of PTPRZ1 in cisplatin resistance of OC and their possible mechanism to provide new thoughts for clinical diagnosis and treatment of OC.

\section{Method}

\section{GEPIA database}

The expression of mRNA profile was analyzed in 426 OC tissues and 88 normal control tissues obtained from TCGA database. GEPIA is a new interactive website for the analysis of RNA sequence data based on TCGA and GTEx (http://gepiacancer-pku.cn/index.html). PTPRZ1 expression in OC tissues and normal control tissues was analyzed. The association of PTPRZ1 expression with the overall survival (OS) and disease free survival (DFS) of OC patients was calculated by GEPIA database.

\section{Sample collection}

Thirty pairs of OC tissues and normal control tissues were collected. All OC patients received no chemotherapy or radiotherapy before surgery. Pathological classification and tumor staging were conducted according to the cancer staging criteria of Union for International Cancer Control. This study protocol was approved by the ethics committee of our hospital. All patients signed the informed consent. This study was performed following the Declaration of Helsinki.

\section{Cell culture}

Ovarian cancer cell lines SKOV3 and A2780 were commercially acquired from the Shanghai Institute of Biochemistry and Cell Biology, CAS (Shanghai, China). Cells were cultured in Dulbecco modified Eagle's culture medium containing 10\% fetal bovine serum (Gibco, Carlsbad, California) in an incubator with $5 \%$ carbon dioxide at $37^{\circ} \mathrm{C}$. Drug-resistant cell lines SKOV3 and A2780 were constructed by treating the proliferated cell cultures using DDP (Meilun Biotech, Dalian, China) at a concentration of $8 \mu \mathrm{M}$ for consecutive 12 weeks.

\section{Cell transfection}

A total of $5 \times 10^{\wedge 4} / \mathrm{ml}$ SKOV3 and A2780 cells (or SKOV3/DDP and A2780/DDP) were seeded into 6-well plate, and the transfection was performed at a cell density of about $70 \%$ by reference to the instructions for use of Lipofectamine 3000 (Invitrogen, USA). Cells were transfected by using PTPRZ1 overexpression 
plasmids and corresponding negative references. Above transfection reagents and corresponding negative references were designed and synthesized by GenePharma (Shanghai, China). Cells were collected for subsequent experiments after $48 \mathrm{~h}$ of transfection.

\section{QRT-PCR}

RNA cells or tissues to be extracted were added into TRIzol reagent (Invitrogen, CA, USA) as per the instructions for use. Post chloroform extraction, the aqueous phase was transferred into a new tube. Isopropanol was used to subside RNAs in the aqueous phase. RNA sediments were washed by using 75\% ethyl alcohol and dried at room temperature. DEPC water was then added for resuspension. RNAs were subject to reverse transcription into cDNAs by using PrimeScript RT reagent Kit (TAKARA, Code No. RR036A) according to its instructions for use. For qRT-PCR, SYBR® Green Master Mix (TaKaRa) was used for the detection on Roche480 as per the instructions for use. GAPDH was used as the internal reference, and the calculation was performed with $2^{-\triangle \triangle C T}$ method. The primer sequence is presented below: PTPRZ1 forward: GCCTGGATTGGGCTAATGGAT, PTPRZ1 reverse: CAGTGCTCCTGTATAGGACCA; GAPDH forward: GGAGCGAGATCCCTCCAAAAT, GAPDH reverse: GGCTGTTGTCATACTTCTCATGG.

\section{MTT experiment}

Cells were seeded into the 96 -well plate at $4 \times 14^{3}$ cells per well. DDP at the concentration of $0 \mu \mathrm{M}, 1 \mu \mathrm{M}$, $2 \mu \mathrm{M}, 5 \mu \mathrm{M}, 10 \mu \mathrm{M}, 20 \mu \mathrm{M}$ and $40 \mu \mathrm{M}$ was added into each group. The plate was then incubated for $48 \mathrm{~h}$ at $37^{\circ} \mathrm{C}$. Subsequently, the culture solution was replaced with new culture medium. With MTT $(0.5 \mathrm{mg} / \mathrm{ml})$ added, the plate was incubated with $5 \% \mathrm{CO}_{2}$ at $37^{\circ} \mathrm{C}$ for $4 \mathrm{~h}$. The culture solution was then carefully removed, and $150 \mu \mathrm{L}$ DSO was added into each well to fully dissolve the generated formazan crystals. The microplate reader was used for absorbance measurement at the wavelength of $570 \mathrm{~nm}$. The experiment was repeated in triplicate.

\section{Cell apoptosis experiment}

After cell transfection, with SKOV3/DDP and A2780/DDP cells collected and washed with PBS, cells were stained by using Annexin V-FITC kit (Beyotime, China) according to the instructions for use. FACSCalibur Flow Cytometer (BD Bioscience, Franklin Lakes, NJ, USA) was then utilized to analyze the cell apoptosis rate.

\section{Western blot}

Transfected SKOV3/DDP and A2780/DDP cells were collected. Upon protein extraction, cell lysis solution containing protease inhibitor PMSF (Beyotime, Nantong, China) was added. On the ice, the supernatant was collected post centrifugation. The protein concentration was detected by using BCA Protein Quantitation Kit (Beyotime, Nantong, China) as per its operating instructions. For albuminous degeneration, cells were heated at $100^{\circ} \mathrm{C}$ after adding SDS-PAGE protein loading buffer. The transfer membrane was conducted after the loading buffer was used up. Corresponding size of PVDF membrane 
cut as per the molecular weight was subject to antigen blocking in $5 \%$ skim milk powder blocking buffer. The incubation was conducted by adding primary antibodies. Another incubation was then performed by using secondary antibodies for subsequent exposure.

\section{Data analysis}

The data were analyzed with SPSS 20.0 and GraphPad Prism 6.0 statistical software. The measurement data were shown as the mean \pm standard deviation. Two sample t-test was used as the statistical method for between-group comparison. $\mathrm{P}<0.05$ was considered statistically significant.

\section{Results}

\section{PTPRZ1 expression was reduced in OC}

The analysis of OC tissues and normal control tissues through GEPIA database found that PTPRZ1 expression was reduced in OC tissues (Figure 1A). The correlation of PTPRZ1 expression with OS and DFS of OC patients was then analyzed via TCGA database, which showed no significant correlation between PTPRZ1 and OS or DFS (Figure 1B, C). Subsequently, qRT-PCR was conducted for PTPRZ1 expression in thirty $\mathrm{OC}$ tissues and thirty control tissues and revealed significant reduction of PTPRZ1 expression in OC tissues (Figure 1D). Moreover, PTPRZ1 expression in DDP-resistant OC tissues SKOV3/DDP and A2780/DDP was significantly lower than that in normal OC cells (Figure 1E).

\section{PTPRZ1 overexpression made OC cells sensitive to cisplatin-induced cytotoxicity}

PTPRZ1 expression was increased by overexpression plasmids in OC cells SKOV3 and A2780, and transfection efficiency was detected through qRT-PCR (Figure 2A). Subsequently, different concentrations of cisplatin were used to treat the transfected cells. The results suggested that PTPRZ1 overexpression made OC cells sensitive to cisplatin-induced cytotoxicity (Figure 2B). The analysis revealed that PTPRZ1 overexpression could considerably reduce the half-maximal inhibitory concentration (IC50) of cisplatin for OC (Figure 2C).

\section{PTPRZ1 negatively regulated DDP sensitivity of OC cells}

The biological effects of PTPRZ1 were further verified in DDP-resistant OC cells SKOV3/DDP and A2780/DDP. Similarly, PTPRZ1 expression in cells was firstly increased with PTPRZ1 overexpressed plasmids (Figure 3A). After transfected SKOV3/DDP and A2780/DDP cells were treated with different concentrations of cisplatin, MTT experiment uncovered that the transfection with oe-PTPRZ1 in cells enhanced the cisplatin sensitivity of cells (Figure 3B) and reduced the IC50 of cisplatin for OC relative to the control group (Figure 3C).

\section{PTPRZ1 overexpression suppressed PI3K/AKT/mTOR pathway}


After transfection with oe-NC and oe-PTPRZ1 in DDP-resistant OC cells SKOV3/DDP and A2780/DDP, respectively, the phosphorylation level of AKT and mTOR proteins was detected by Western blot. The results revealed that PTPRZ1 overexpression in SKOV3/DDP and A2780/DDP cells could decrease p-AKT and p-mTOR protein expression. Additionally, PTPRZ1 exerted its biological effects by suppressing $\mathrm{PI} 3 \mathrm{~K} / \mathrm{AKT} / \mathrm{mTOR}$ pathway (Figure 4).

\section{The activation of PI3K/AKT/mTOR pathway reversed PTPRZ1 overexpression mediated pro-apoptotic effects}

To further verify whether PTPRZ1 involved in the cisplatin resistance process of OC via regulating $\mathrm{PI3K} / \mathrm{AKT} / \mathrm{mTOR}$ pathway, with PTPRZ1 overexpression and transfection with PI3K/AKT/mTOR agonist IGF-1 in SKOV3/DDP and A2780/DDP cells, cell apoptosis was detected using a flow cytometer. In Figure 5A, after PTPRZ1 was overexpressed in SKOV3/DDP and A2780/DDP cells, notably higher proportion of cell apoptosis was observed; this proportion was reduced post transfection with IGF-1. Furthermore, PTPRZ1 overexpression enhanced the protein level of $C$ caspase and BAX and suppressed the protein level of BCL-2; with concurrent transfection with IGF-1, lower protein level of $C$ caspase and BAX and higher protein level of BCL-2 were observed (Figure 5B). These findings objectivized that PTPRZ1 regulated the chemosensitivity of cisplatin to $\mathrm{OC}$ cells by regulating PI3K/AKT/mTOR pathway.

\section{Discussion}

$\mathrm{OC}$ is susceptible to difficult early diagnosis, chemoresistance and recurrence, with 5-year survival rate of only about $40 \%$, thus it has been a hotspot among clinicians(12). Main causes for low survival rate of OC include low early diagnosis and susceptibility to recurrence or metastasis. Previous studies showed that in addition to unique characteristics of $\mathrm{OC}$, less sensitivity of $\mathrm{OC}$ cells to chemotherapeutic drugs was also a cause for recurrence, metastasis and then failure of tumor treatment $(13,14)$. Since targeted therapy is a means for tumor treatment by selective inhibition of molecular pathway, a question is raised that whether selective targeted therapy may be provided for the resistance mechanism. At present, there is no available clinical trial to demonstrate the ideal response rate of targeted therapy. Neither singletarget therapy nor combination with current chemotherapy regimens improves the drug resistance or significantly increase the patients' survival rate.

Early bioinformatic analysis and qRT-PCR detection revealed notably low expression of PTPRZ1 in OC. For this reason, we made further study. The reason why cisplatin was selected as the study subject for drug resistance was that platinum drugs had become indispensable drugs for OC chemotherapy. It exerted cytotoxic effects in cells and had chemotherapy effects. The specific mechanism preliminarily confirmed a possible association with apoptosis process $(15,16)$. Cisplatin is the first-line chemotherapeutic drug for $\mathrm{OC}$, and there is a close association of platinum resistance with postoperative survival of OC patients $(17,18)$. PTPRZ1 expression was considerably reduced in DDP-resistant OC cell lines as detected. In vitro cell experiments suggested that PTPRZ1 overexpression made OC cells sensitive to cisplatin-induced cytotoxicity and considerably reduced the IC50 of OC for cisplatin. 
Furthermore, we explored and verified the possible molecular mechanisms of PTPRZ1. In Western blot, overexpression of PTPRZ1 suppressed the phosphorylation level of AKT and mTOR, suggesting that PTPRZ1 participated in the cisplatin resistance of OC possibly through inhibiting PI3K/AKT/mTOR pathway. PI3K/AKT/mTOR pathway is a signal transduction pathway extensively distributed in cells to involve in cell growth, suppress cell apoptosis and maintain important functions of cells $(19,20)$. P13K may be activated by multiples factors including Insulin-like growth factor (IGF-1) to activate the downstream AKT via phosphorylation. Activated AKT may phosphorylate tuberous sclerosis complex 2 and attenuate the inhibiting effect of TSC2 on its downstream mTOR(21-23). MTOR is an intracellular serine-threonine kinase that is highly conservative for its evolution. It is widely expressed in various biological cells. The activation of mTOR phosphorylates the downstream P70S6K1 effector to initiate the translation process and promote the synthesis of RNA and proteins $(24,25)$. This pathway participates in the occurrence and development of multiple tumors and angiogenesis, and is also considered as the primary pathway for cancer cell survival $(26,27)$. Then, in vitro cell experiment indicated that the transfection with PI3K/AKT/mTOR pathway agonist IGF-1 recovered the pro-apoptotic effects of PTPRZ1 overexpression.

Meanwhile, this study also had many limitations. Firstly, no in vivo experiment was performed to verify the biological effects and regulatory mechanisms of PTPRZ1; secondly, other downstream targets of PTPRZ1 should be explored and verified in future studies. Taken together, this study initially confirmed that PTPRZ1 suppressed the cisplatin resistance of OC and induced the cytotoxicity by blocking $\mathrm{PI} 3 \mathrm{~K} / \mathrm{AKT} / \mathrm{mTOR}$ pathway. This provides new perspective and theoretical basis for clinical treatment of OC.

\section{Conclusion}

This study made preliminary discussion of the roles of PTPRZ1 in cisplatin resistance of OC and their possible mechanism, and provided new thoughts for clinical treatment of OC.

\section{Declarations}

\section{Acknowledgements}

Not applicable.

\section{Authors' contributions}

PW, YJH, PPQ and BHK conceived and designed this study. PW, YZ, JL and JGZ helped with data collection and summary. PW, YJH, PPQ, YZ, JL and BHK were responsible for data analysis and interpretation. All authors made contributions to manuscript writing. The authors read and approved the final manuscript.

\section{Funding}


Not applicable.

\section{Availability of data and materials}

The datasets used and/or analyzed during the present study are available from the corresponding author on reasonable request.

\section{Ethics approval and consent to participate}

The study was approved by the Ethics Committee of Qilu Hospital,Cheeloo College of Medicine,Shandong University.

\section{Consent for publication}

Not applicable.

\section{Competing interests}

The authors declare that they have no competing interests.

\section{Author details}

${ }^{1}$ Qilu Hospital,Cheeloo College of Medicine,Shandong University, Jinan 250012, People's Republic of China. ${ }^{2}$ Tianjin Central Hospitalof Obstetrics and Gynecology, Tianjin 300000, People's Republic of China.

\section{References}

1. Mirza MR, Coleman RL, Gonzalez-Martin A, Moore KN, Colombo N, Ray-Coquard I, et al. The forefront of ovarian cancer therapy: update on PARP inhibitors. Ann Oncol. 2020.

2. Trimbos JB. Surgical treatment of early-stage ovarian cancer. Best Pract Res Clin Obstet Gynaecol. 2017;41:60-70.

3. van Jaarsveld MT, Helleman J, Boersma AW, van Kuijk PF, van ljcken WF, Despierre E, et al. miR-141 regulates KEAP1 and modulates cisplatin sensitivity in ovarian cancer cells. Oncogene. 2013;32(36):4284-93.

4. Bogani G, Lopez S, Mantiero M, Ducceschi M, Bosio S, Ruisi S, et al. Immunotherapy for platinumresistant ovarian cancer. Gynecol Oncol. 2020.

5. Lund RJ, Huhtinen K, Salmi J, Rantala J, Nguyen EV, Moulder R, et al. DNA methylation and Transcriptome Changes Associated with Cisplatin Resistance in Ovarian Cancer. Sci Rep. 2017;7(1):1469.

6. Li X, Chen W, Jin Y, Xue R, Su J, Mu Z, et al. miR-142-5p enhances cisplatin-induced apoptosis in ovarian cancer cells by targeting multiple anti-apoptotic genes. Biochem Pharmacol. 2019;161:98- 
112.

7. Islam SS, Aboussekhra A. Sequential combination of cisplatin with eugenol targets ovarian cancer stem cells through the Notch-Hes1 signalling pathway. J Exp Clin Cancer Res. 2019;38(1):382.

8. Rada M, Nallanthighal S, Cha J, Ryan K, Sage J, Eldred C, et al. Inhibitor of apoptosis proteins (IAPs) mediate collagen type XI alpha 1-driven cisplatin resistance in ovarian cancer. Oncogene. 2018;37(35):4809-20.

9. Matjasic A, Zupan A, Bostjancic E, Pizem J, Popovic M, Kolenc D. A novel PTPRZ1-ETV1 fusion in gliomas. Brain Pathol. 2020;30(2):226-34.

10. Huang P, Ouyang DJ, Chang S, Li MY, Li L, Li QY, et al. Chemotherapy-driven increases in the CDKN1A/PTN/PTPRZ1 axis promote chemoresistance by activating the NF-kappaB pathway in breast cancer cells. Cell Commun Signal. 2018;16(1):92.

11. Shang $D$, Xu X, Wang D, Li Y, Liu Y. Protein tyrosine phosphatase zeta enhances proliferation by increasing beta-catenin nuclear expression in VHL-inactive human renal cell carcinoma cells. World $\mathrm{J}$ Urol. 2013;31(6):1547-54.

12. Langdon SP, Herrington CS, Hollis RL, Gourley C. Estrogen Signaling and Its Potential as a Target for Therapy in Ovarian Cancer. Cancers (Basel). 2020;12(6).

13. Yang YI, Ahn JH, Choi YS, Choi JH. Brown algae phlorotannins enhance the tumoricidal effect of cisplatin and ameliorate cisplatin nephrotoxicity. Gynecol Oncol. 2015;136(2):355-64.

14. Tanenbaum LM, Mantzavinou A, Subramanyam KS, Del Carmen MG, Cima MJ. Ovarian cancer spheroid shrinkage following continuous exposure to cisplatin is a function of spheroid diameter. Gynecol Oncol. 2017;146(1):161-9.

15. Xiao L, Peng Z, Zhu A, Xue R, Lu R, Mi J, et al. Inhibition of RUNX1 promotes cisplatin-induced apoptosis in ovarian cancer cells. Biochem Pharmacol. 2020;180:114116.

16. Guo X, Fang Z, Zhang M, Yang D, Wang S, Liu K. A Co-Delivery System of Curcumin and p53 for Enhancing the Sensitivity of Drug-Resistant Ovarian Cancer Cells to Cisplatin. Molecules. 2020;25(11).

17. Zhang X, Wang LL, Wang B, Liu HL, Zhang J, Li YH, et al. Effect of siRNA-induced Atg7 gene silencing on the sensitivity of ovarian cancer SKOV3 cells to cisplatin. Am J Transl Res. 2020;12(5):2052-61.

18. Zampieri LX, Grasso D, Bouzin C, Brusa D, Rossignol R, Sonveaux P. Mitochondria participate in chemoresistance to cisplatin in human ovarian cancer cells. Mol Cancer Res. 2020.

19. Chen K, Shang Z, Dai AL, Dai PL. Novel PI3K/Akt/mTOR pathway inhibitors plus radiotherapy: Strategy for non-small cell lung cancer with mutant RAS gene. Life Sci. 2020;255:117816.

20. Han Y, Wang J, Wang Z, Xu B. Comparative efficacy and safety of CDK4/6 and PI3K/AKT/mTOR inhibitors in women with hormone receptor-positive, HER2-negative metastatic breast cancer: a systematic review and network meta-analysis. Curr Probl Cancer. 2020:100606. 
21. Wanigasooriya K, Tyler R, Barros-Silva JD, Sinha Y, Ismail T, Beggs AD. Radiosensitising Cancer Using Phosphatidylinositol-3-Kinase (PI3K), Protein Kinase B (AKT) or Mammalian Target of Rapamycin (mTOR) Inhibitors. Cancers (Basel). 2020;12(5).

22. Braglia L, Zavatti M, Vinceti M, Martelli AM, Marmiroli S. Deregulated PTEN/PI3K/AKT/mTOR signaling in prostate cancer: Still a potential druggable target? Biochim Biophys Acta Mol Cell Res. 2020;1867(9):118731.

23. Huang TT, Lampert EJ, Coots C, Lee JM. Targeting the PI3K pathway and DNA damage response as a therapeutic strategy in ovarian cancer. Cancer Treat Rev. 2020;86:102021.

24. Nepstad I, Hatfield KJ, Gronningsaeter IS, Reikvam H. The PI3K-Akt-mTOR Signaling Pathway in Human Acute Myeloid Leukemia (AML) Cells. Int J Mol Sci. 2020;21(8).

25. Sobocan M, Bracic S, Knez J, Takac I, Haybaeck J. The Communication Between the $\mathrm{PI} 3 \mathrm{~K} / \mathrm{AKT} / \mathrm{mTOR}$ Pathway and Y-box Binding Protein-1 in Gynecological Cancer. Cancers (Basel). 2020;12(1).

26. Xu F, Na L, Li Y, Chen L. Roles of the PI3K/AKT/mTOR signalling pathways in neurodegenerative diseases and tumours. Cell Biosci. 2020;10:54.

27. Mirza-Aghazadeh-Attari M, Ekrami EM, Aghdas SAM, Mihanfar A, Hallaj S, Yousefi B, et al. Targeting PI3K/Akt/mTOR signaling pathway by polyphenols: Implication for cancer therapy. Life Sci. 2020;255:117481.

\section{Figures}

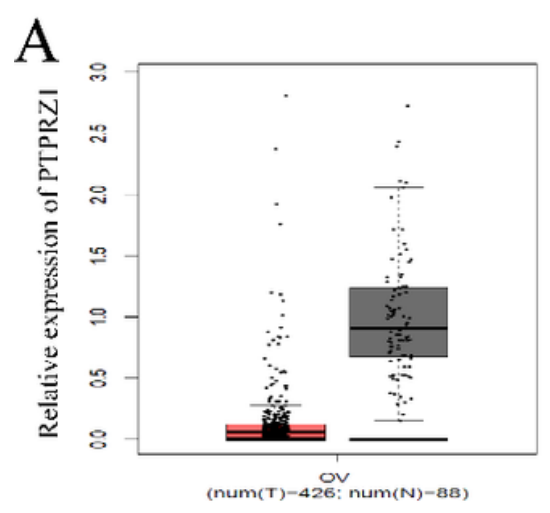

$\mathrm{D}$

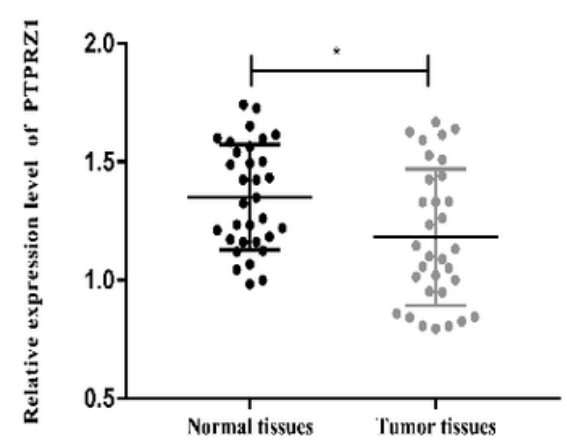

B

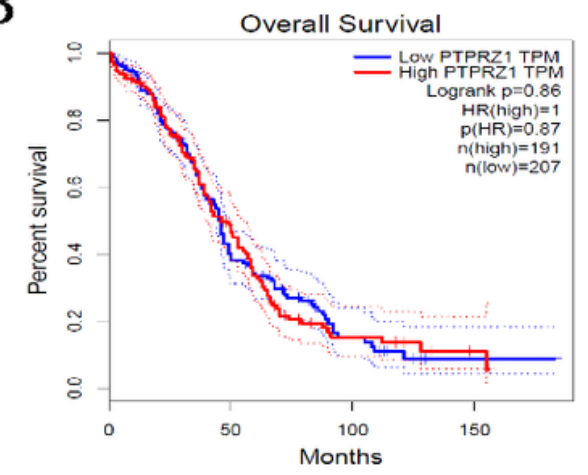

$\mathrm{E}$

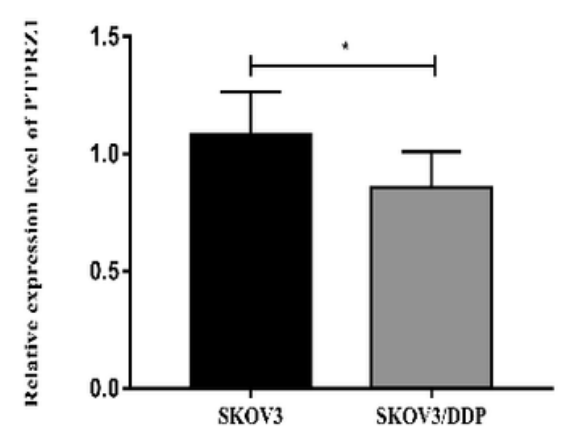

C
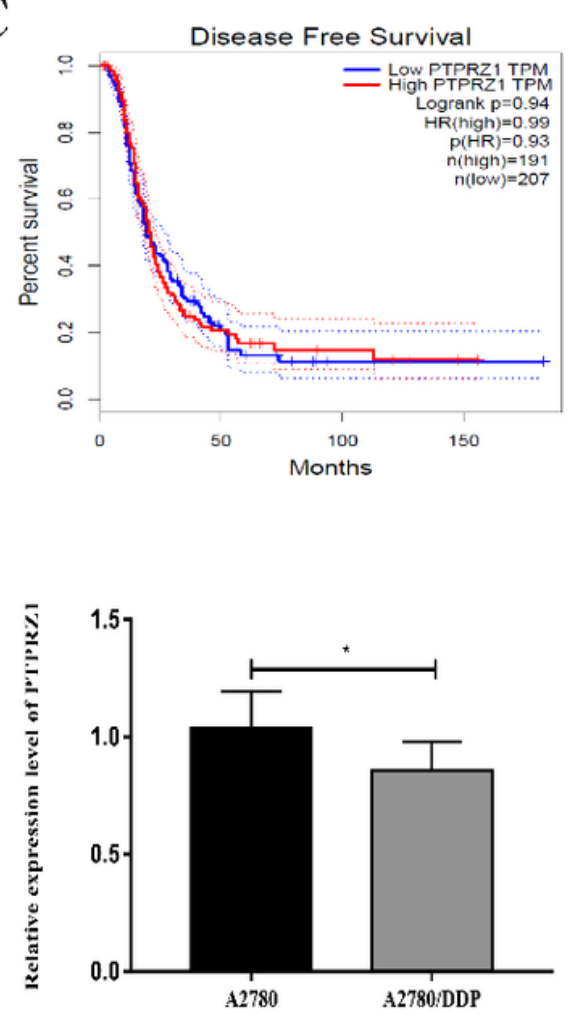
Figure 1

PTPRZ1 expression was reduced in OC A. PTPRZ1 expression in OC tissues and normal control tissues as analyzed by GEPIA database. B. The analysis of TCGA database uncovered no considerable association of PTPRZ1 expression with OS of OC patients. C. The analysis of TCGA database revealed no remarkable correlation of PTPRZ1 expression with DFS of OC patients. D. Relative expression of PTPRZ1 in OC tissues and normal control tissues by qRT-PCR. E. Lower PTPRZ1 expression in DDPresistant OC cells relative to normal OC cells. Data was expressed as mean \pm SD. ${ }^{*} p<0.05$
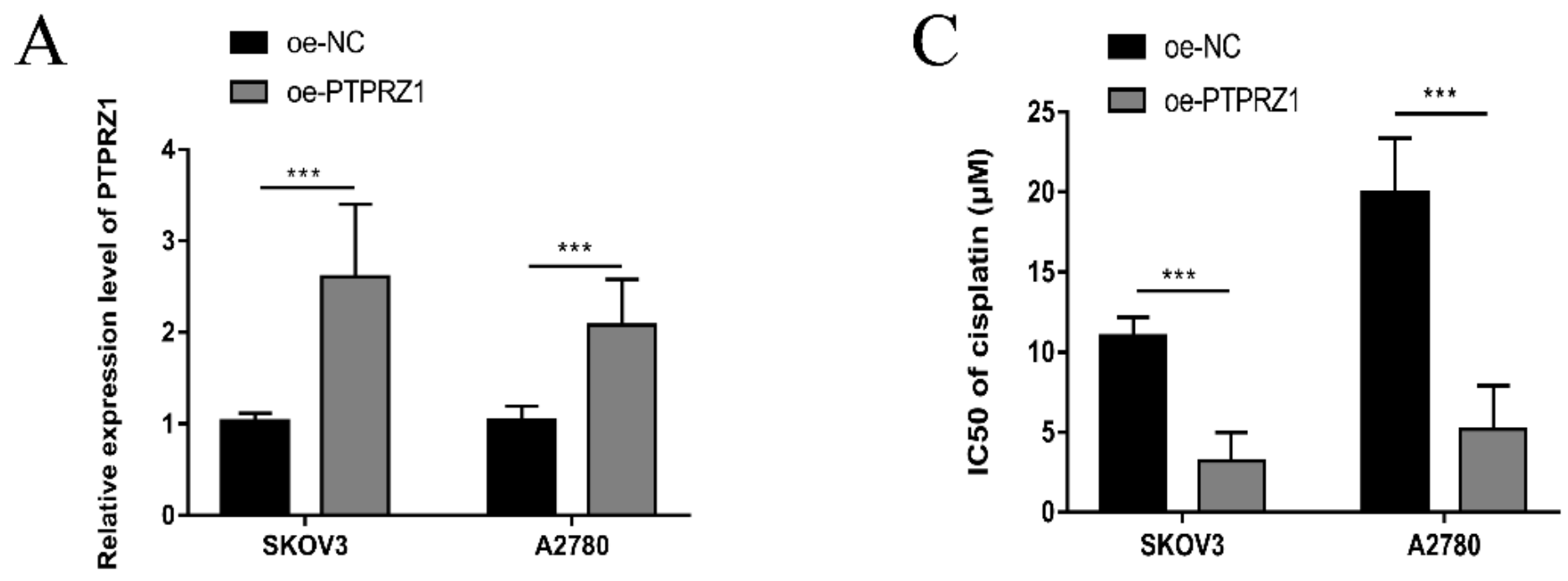

$\mathrm{B}$

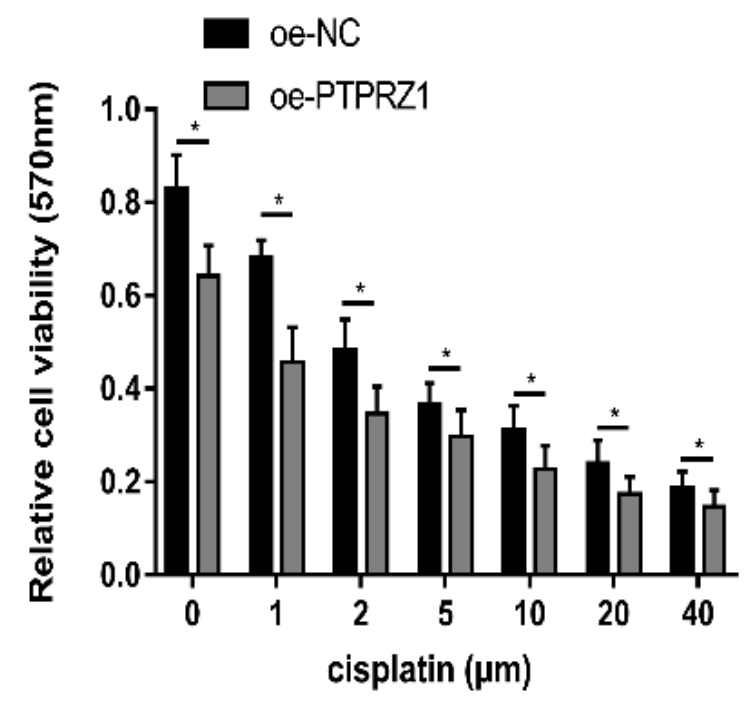

SKOV3

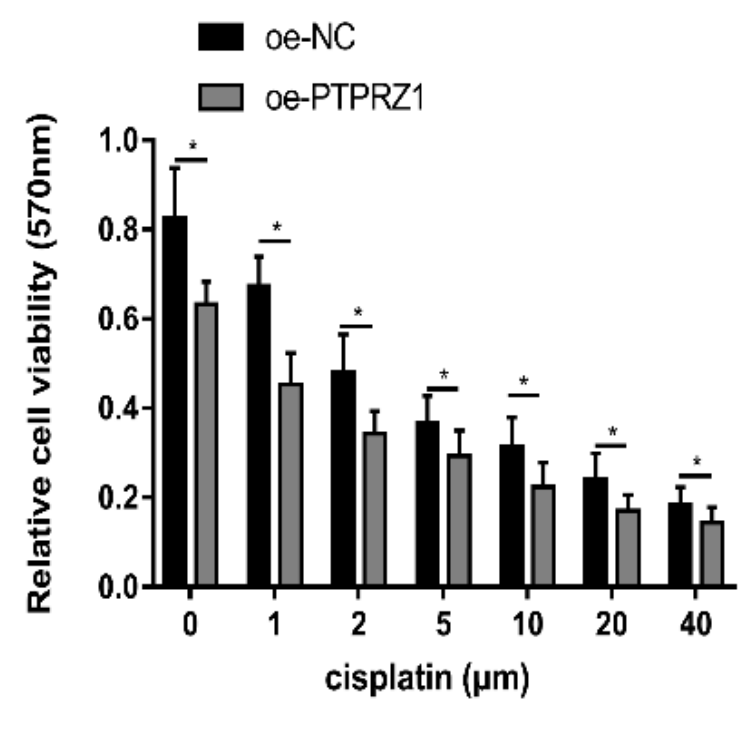

A2780

Figure 2

PTPRZ1 overexpression made OC cells sensitive to cisplatin-induced cytotoxicity A. Transfection efficiency of PTPRZ1 overexpression plasmids in OC cells SKOV3 and A2780 by qRT-PCR. B. The cytotoxicity of different concentrations of cisplatin was increased in cells lines SKOV3 and A2780 by 
transfection with PTPRZ1 overexpression plasmids. C. Effects of PTPRZ1 on IC50 of cisplatin in cell lines SKOV3 and A2780. Data was expressed as mean \pm SD. ${ }^{*} p<0.05 ; * \star * p<0.001$
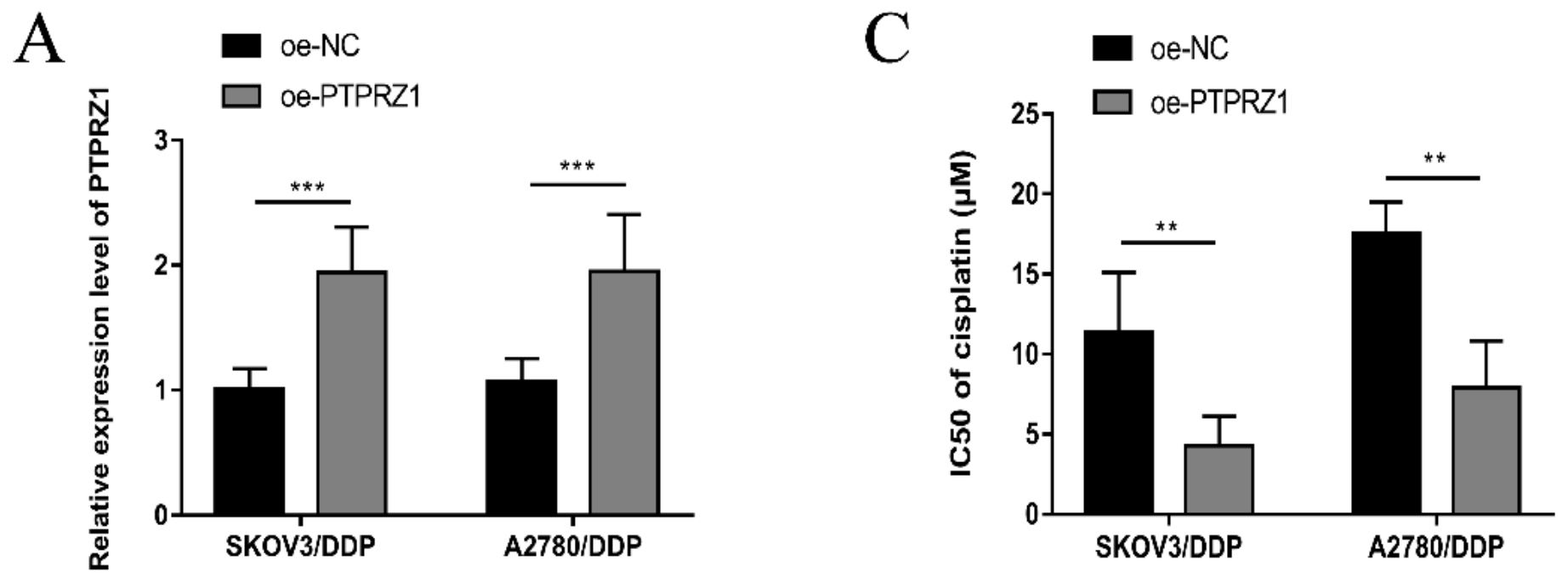

$\mathrm{B}$
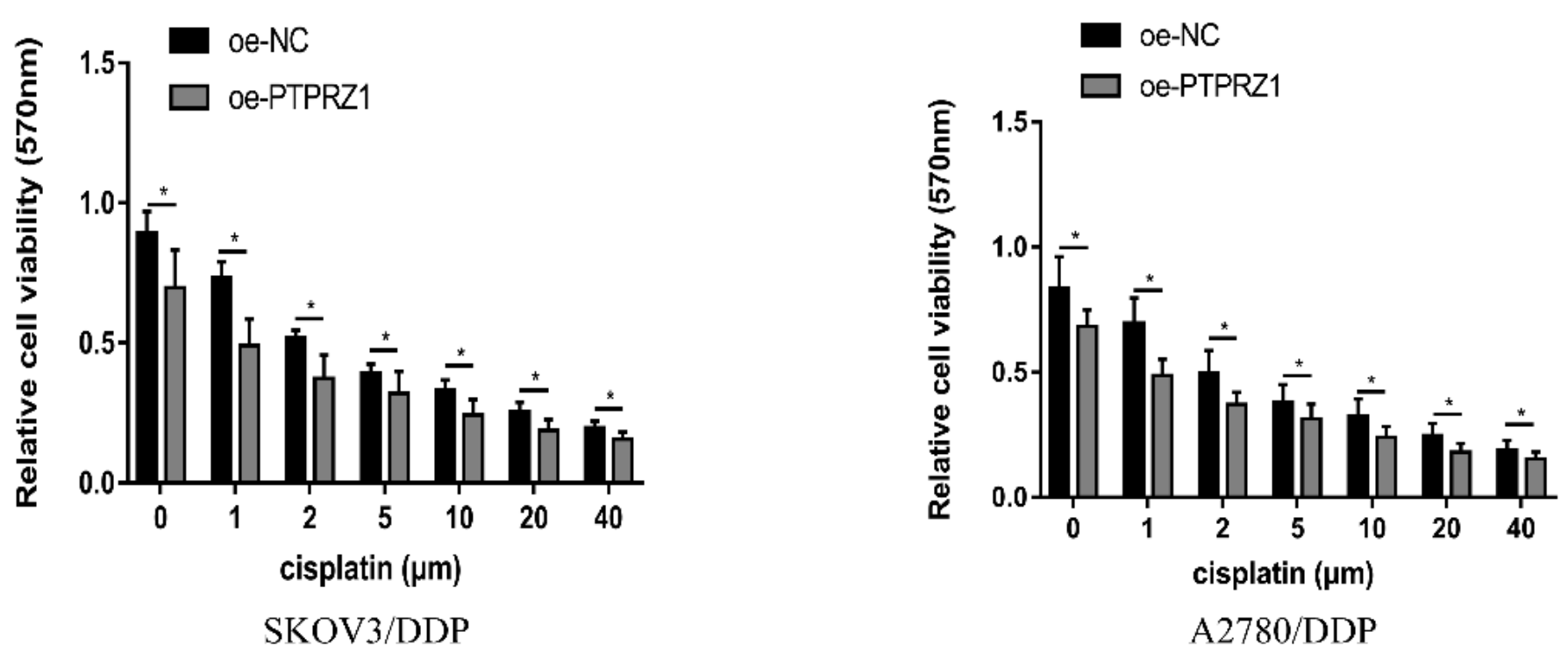

Figure 3

PTPRZ1 negatively regulated DDP sensitivity of OC cells A. Transfection efficiency of PTPRZ1 overexpression plasmids in DDP-resistant OC cells SKOV3/DDP and A2780/DDP by qRT-PCR. B. The cytotoxicity of different concentrations of cisplatin was enhanced in cell lines SKOV3/DDP and A2780/DDP by transfection with PTPRZ1 overexpression plasmids. C. Effects of PTPRZ1 on IC50 of cisplatin in cell lines SKOV3/DDP and A2780/DDP. Data was expressed as mean \pm SD. ${ }^{\star} p<0.05 ;{ }^{\star \star} p<0.01$; $\star * \star p<0.001$. 


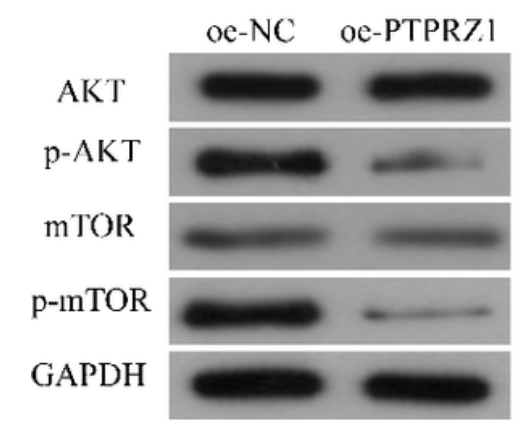

SKOV3/DDP

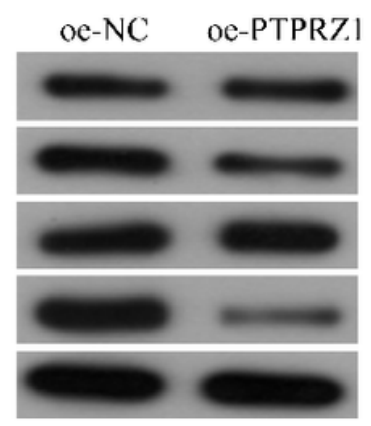

A2780/DDP

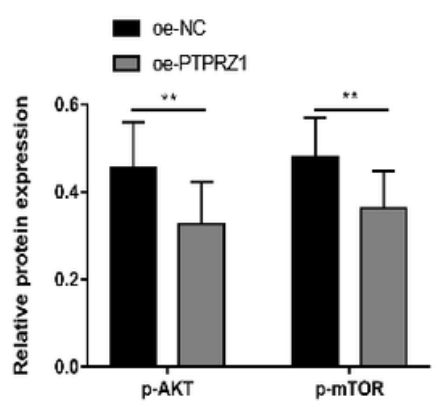

SKOV3/DDP

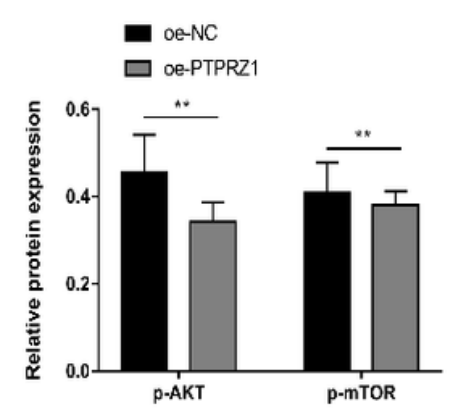

A2780/DDP

\section{Figure 4}

PTPRZ1 regulated PI3K/AKT/mTOR pathway in cell lines SKOV3/DDP and A2780/DDP Phosphorylation level of AKT and mTOR detected by Western blot experiment post overexpression of PTPRZ1 in cell lines SKOV3/DDP and A2780/DDP. Data was expressed as mean \pm SD. ${ }^{* *} \mathrm{p}<0.01$.

A
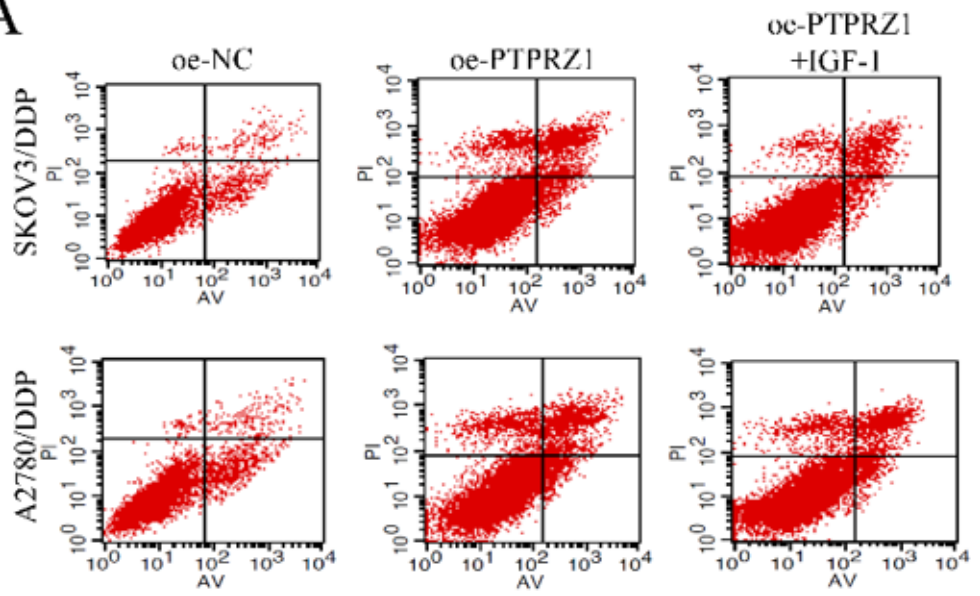

B

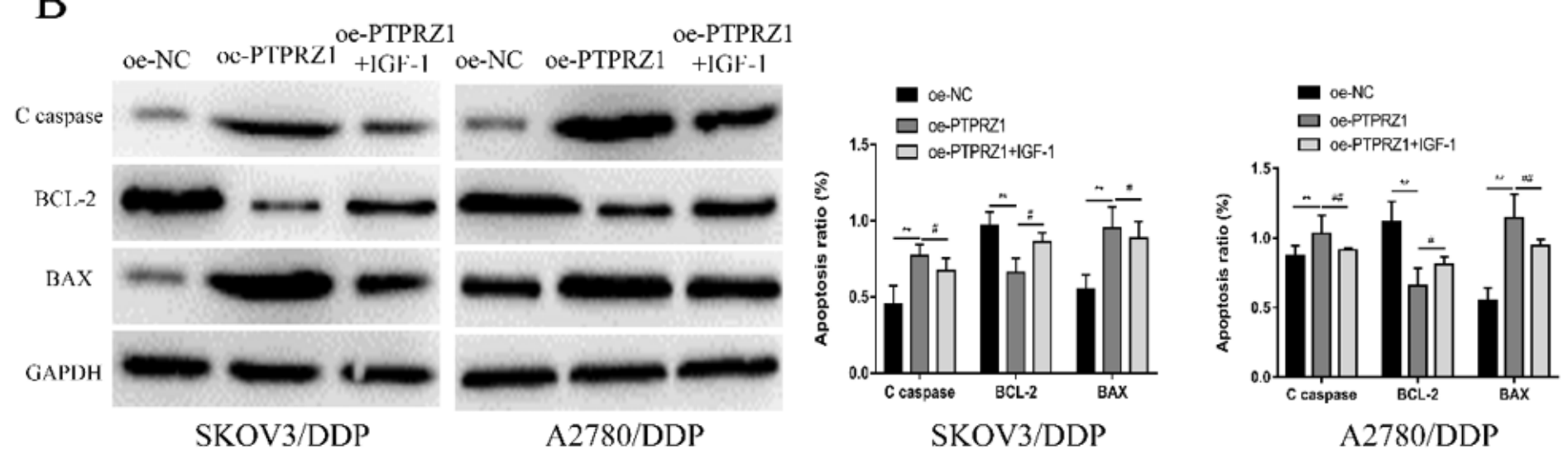

Figure 5

The activation of PI3K/AKT/mTOR pathway reversed PTPRZ1 overexpression mediated pro-apoptotic effects A. Apoptosis of SKOV3/DDP and A2780/DDP cells post treatment with PTPRZ1 overexpression plasmids and PI3K/AKT/mTOR agonist IGF-1 by flow cytometry. B. Protein level of C caspase, BAX and 
BCL-2 in SKOV3/DDP and A2780/DDP cells post treatment with PTPRZ1 overexpression plasmids and $\mathrm{PI} 3 \mathrm{~K} / \mathrm{AKT} / \mathrm{mTOR}$ agonist IGF-1 by Western blot experiment. Data was expressed as mean $\pm \mathrm{SD}$. ${ }^{\star \star} \mathrm{p}<0.01$; $\star \star \star p<0.001, \# p<0.05 ; \# \#<<0.01$. 